\title{
Surgical approach to adrenal ganglioneuroma: Case report and literature review
}

\author{
Danilo Abate, Giuseppe Giusti, Nicola Caria, Marco Lucci Chiarissi, Antonello De Lisa \\ Clinica Urologica, Università degli Studi di Cagliari, Ospedale SS. Trinità, Cagliari, Italy.
}

\begin{abstract}
Summary Objective: Ganglioneuroma $(G N)$ is a benign tumor with a slow growth that can originate from any paravertebral sympathetic plexus. It is usually asymptomatic or with nonspecific symptoms. TC and RM scan are helpful to study GN. It is usually represented by an ovalshaped retroperitoneal mass or, in case of adrenal impairment, by low radiologic contrast media attenuation. Surgical treatment is mandatory. Literature shows how the laparoscopic approach is the most used, especially in lesions that are $6 \mathrm{~cm}$ or smaller. Our purpose is to describe our experience on an incidental adrenal GN of about $5 \mathrm{~cm}$ treated by the laparoscopic transperitoneal approach.

Materials and methods: A 33-year-old male had ultrasound occasional finding of an about $4 \mathrm{~cm}$ adrenal mass. TC and RM scan identified a retroperitoneal mass (max diameter $48 \mathrm{~mm}$ ). The lesion was removed with a transperitoneal laparoscopic approach.

Results: No intraoperative or postoperative complications occurred. The patient was discharged 3 days after surgery. Conclusions: Up to the present laparoscopic surgery is the best approach for GN treatment.
\end{abstract}

KEY WORDS: Adrenal ganglioneuroma.

Submitted 17 April 2018; Accepted 29 April 2018

\section{INTRODUCTION}

Ganglioneuroma (GN) is a benign tumor with a slow growth that can originate from any paravertebral sympathetic plexus but can occasionally develop in the adrenal medulla. It is usually asymptomatic or with nonspecific symptoms related to mass effect causing diaphragmatic compression, upper urinary tract or gastrointestinal upset and, rarely, spinal cord compression or bone erosions. Sometimes GN produces catecholamines or other hormones so patients can have hypertension, diarrhea or flushing.

TC and RM scan are helpful to study GNs. They are usually represented by an oval-shaped retroperitoneal mass or, in case of adrenal impairment, by low radiologic contrast media attenuation. RM study is characterized by signal hypointensity during T-1 weighted scans and a non uniform hyperintensity in T-2 weighted scans (1). From the histopathologic point of view, GN presents mature Schwann cells, ganglion cells and nerve fiber (2). Surgical treatment is mandatory. Literature shows how the laparoscopic approach is the most used, especially in less than $6 \mathrm{~cm}$ lesions.

The majority of authors consider transperitoneal approach as best solution because it guarantees a good retroperitoneal access and maximum adrenal and big vessels exposition (3). According to this technique, with a patient in right lateral posture, Zofragos et al. have completed right adrenalectomy in a 33-year-old female with a $13 \mathrm{~cm}$ GN, whereas Abraham et al. have performed the same surgery in a 33-year-old female with a $17 \mathrm{~cm}$ GN. Low intraoperative blood loss and no postoperative complications occurred in both studies.

The open approach should be preferred when the tumor is close to big vessels or other organs.

\section{Case description}

A 33-year-old male was hospitalized in March 2016 for urinary calculi. During ultrasound abdominal study, we occasionally found a $40 \mathrm{~mm}$ left adrenal mass.

In November 2016 he underwent TC abdomen scan with radiological contrast media. The lesion was an ovalshaped gross and homogeneous mass of about $46 \mathrm{~mm}$. Its radiological aspect was compatible with low adipose tissue adenoma (median density was $45 \mathrm{HU}$ ).

On 3 January 2017 he underwent an abdomen RM without radiological contrast media and the left adrenal lesion (48 x $30 \mathrm{~mm}$ ) was featured by low hyperintensity in T-2 weighted scans and signal hypointensity during T1 weighted ones. In the beginning, the mass was described as low lipidic adenoma. Plasmatic values of cortisol, aldosterone, renin, and TSH were in range and plasmatic ACTH was not suppressed. Aldosterone/renin ratio was 4,86 pg/ml. Daily urinary metanephrine and normetanephrine were normal. We performed laparoscopic transperitoneal adrenalectomy. The patient was placed in right lateral posture. Pneumoperitoneum was induced with open Hasson technique. An optical port was placed at the umbilicus, the other 2 trocars were placed respectively in left iliac fossa and in the left pararectal region. The left paracolic gutter was incised and renal loggia was exposed. Then, the anterior membrane of Gerota fascia was incised and renal vascular hilum isolated. An accurate craniocaudal dissection was performer till the adrenal gland exposition. Adrenal ves- 
sels were isolated and adrenal vein was cut between 3 Hem-o-Lok clips just at the confluence with the renal vein. The adrenal artery was cut by a vessel sealing device. Then, adrenalectomy was completed and adrenal gland was extracted into $10 \mathrm{~mm}$ Endo-bag. No drainage was placed. A urinary catheter was placed and then removed after 24 hours.

Routine blood samplings were taken. The difference between pre-surgery and 6 hours later hemoglobin values was $0.8 \mathrm{~g} / \mathrm{dl}$.

The patient was discharged 3 days after surgery. Histopathological analysis showed an adrenal ganglioneuroma with isolated mature ganglion cells, packaged in 3-4 elements with no cellular atypia. Immunohistochemistry was positive for S100 and NSE (Figure 1).

\section{Conclusions}

Our case report and a literature review confirm feasibility, efficacy, and safety of surgical management of this disease. A laparoscopic approach can reduce invasivity, blood loss, and hospital stay.

\section{REFERENCES}

1. Ichikawa T, Ohtomo K, Araki T, et al. Ganglioneuroma: computed tomography and magnetic resonance features. Brit J Radiol. 1996; 69:114-121.
Figure 1.

Histology showed "adrenal ganglioneuroma with isolated mature ganglion cells, packaged in 3-4 elements with no cellular atypia". Section 1 highlights in H\&E stain 100x aggregates of ganglion cells, section II low positivity on Ki67 immunohistochemistry, section III \& IV strong positivity on NSE and S100 respectively.

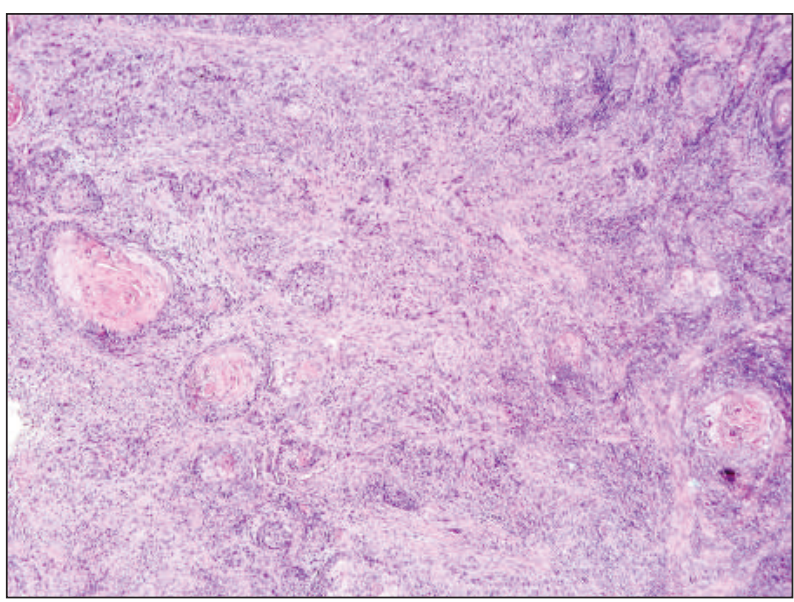

2. Chang CY, Hsieh YL, Hung GY, et al. Ganglioneuroma presenting as an asymptomatic huge posterior mediastinal and retroperitoneal tumor. J Chin Med Assoc. 2003; 66:370-374.

3. Zografos GN, Kothonidis K, Ageli C, et al. Laparoscopic resection of large adrenal ganglioneuroma. J Soc Laparoendoscopic Surg, 2007; 11:487-492.

\section{Correspondence}

Danilo Abate, MD

Giuseppe Giusti, MD (Corresponding Author)

giuseppegiusti.med@gmail.com

Nicola Caria, MD (Corresponding Author)

nicolacarial@gmail.com

Marco Lucci Chiarissi, MD

Antonello De Lisa, MD

Clinica Urologica, Università degli Studi di Cagliari, Ospedale SS. Trinità

Via Is Mirrionis, Cagliari, Italy 